BABELAO 2 (2013), p. 123-139

(C) ABELAO (Belgium)

\title{
Un solo manuscrito en dos bibliotecas: el comentario de Raši a la Biblia
}

Por

\section{$\mathrm{M}^{\mathrm{a}}$ Teresa Ortega Monasterio}

Centro de Ciencias Humanas y Sociales

CSIC, Madrid

$\mathrm{E}$ 1 mundo del patrimonio escrito de los judíos en la Península Ibérica es complejo y extenso. El legado existente en la actualidad en bibliotecas españolas no es grande, pero sí significativo tanto en lo que a calidad textual se refiere, como en cuanto que es reflejo de una sociedad multicultural en la que se produce. En dichas bibliotecas existen manuscritos en caracteres hebreos pertenecientes a un amplio espacio de tiempo, que va desde el siglo XIII hasta el XIX, de materias muy dispares, estando todas comprendidas en el ámbito de los estudios judíos ${ }^{1}$.

\footnotetext{
${ }^{1}$ Sobre las colecciones de manuscritos hebreos en bibliotecas españolas, puede verse M.T. OrTEga MONASTERIO, « Spanish Biblical Hebrew Manuscripts ", Hebrew Studies 45 (2004), p. 163-174; IBID., " Hebrew Collections in Spain: Catalogues and Inventories », in J.P. MONFERRER-SALA - H.G.B. TEULE - S. TORAllas TOVAR (eds.), Eastern Christians and their Written Heritage, (Eastern Christian Studies 14) Louvain, 2012; IBID., "Historia y formación de las colecciones de manuscritos hebreos en España », in E. ALFONSO - J. DEL
} 
Aunque el género más representativo puedan ser las Biblias, tanto por su calidad como por su belleza, podemos afirmar sin temor a equivocarnos que los comentarios bíblicos le siguen en importancia. No hay que olvidar que la Biblia era el eje central de la vida judía desarrollada en territorios peninsulares durante toda la Edad Media y ello da lugar a un sinfín de copias de los comentarios más afamados y de los autores más destacados. Los manuscritos conservados en España que copian comentarios bíblicos son, en su gran mayoría, copias sefardíes, como puede apreciarse en los catálogos existentes ${ }^{2}$. Sin embargo, encontramos algunas excepciones: en la biblioteca de la Abadía de Montserrat existen varios manuscritos de procedencia oriental con comentarios bíblicos y otro de origen italiano. Pero existen además dos manuscritos de origen askenazí que, aunque se conservan en dos bibliotecas distintas, forman parte de una sola unidad como veremos a continuación.

Uno de los numerosos manuscritos que copian el comentario de Raši a la Torah es el que se conserva en dos bibliotecas españolas: la biblioteca del museo Lázaro Galdiano de Madrid ${ }^{3}$ y la Biblioteca Colombina de Sevilla ${ }^{4}$. Y ambas bibliotecas comparten el manuscrito debido a que se encuentra dividido en dos bloques: el primero de ellos contiene el comentario al Pentateuco, además de algunos folios sueltos de los libros de Reyes, Esdras y Crónicas y el segundo contiene el comentario al resto de la biblia hebrea. El estado de conservación de ambos es bueno, aunque presentan algunas diferencias. Mientras que el conservado en el museo Lázaro Galdiano está en excelente estado de conservación, encuadernado en pasta con cortes dorados y lomo con texto en letras doradas con el título $M$. S. Hebreo, el de Sevilla tiene algunos folios de difícil lectura; en el fol. 307v hay una nota manuscrita en la que se indica que Antonio Galván restauró la encuadernación de época en la primavera de 1992 y confeccionó la caja estuche para su conservación ${ }^{5}$. Los cuadernos son en su mayoría seniones excepto el cuaderno 13, que es un ternión, y el último, que es un folio suelto, faltando el resto del cuaderno con el final del texto del manuscrito. La composición de cuadernos del manuscrito es la

Barco - M.T. Ortega Monasterio - A. Prats (eds.), Biblias de Sefarad, Madrid, 2012, p. 149-175.

2 Las catalogaciones más recientes son las realizadas por F.J. DEL BARCO, Catálogo de Manuscritos Hebreos de la Comunidad de Madrid, 3 vols. Madrid, 20032006; IBID., Catálogo de manuscritos hebreos de la Biblioteca de Montserrat, Barcelona, 2008.

${ }^{3}$ Número de inventario 15.646.

4 Signatura 56-1-16. Para una descripción detallada del manuscrito, véase F.J. Del Barco Del BARCo, Catálogo de Manuscritos Hebreos de la Comunidad de Madrid, vol. 2, Madrid, 2004, p. 150-151; F. CANTERA Burgos, « Nueva serie de manuscritos hebreos en Madrid », Sefarad 19 (1959), p. 36-42 y E. ALFONSO - J. Del Barco - M.T. Ortega Monasterio - A. Prats (eds.), Biblias de Sefarad, Madrid, 2012, No Catálogo 47 y 48, p. 369-375.

${ }^{5}$ Se trata de Antonio Galván Cuéllar, junto con su hermano José, prestigiosos encuadernadores artísticos del siglo XX con taller en Cádiz. Por sus manos han pasado valiosos ejemplares de las más importantes bibliotecas españolas. 
siguiente (se indican en negrita los folios que corresponden al documento conservado en el Museo Lázaro Galdiano) ${ }^{6}$ :

1: VI ( $3^{\text {a }}$ guarda inicial + ff. 1, 2-11)

2-5: VI (ff. 12-23, 24-35, 36-47, 48-59).

6: VI (ff. 60-67, 68, 69-71).

7: VI (ff. 72-83).

8: VI (ff. 84-95).

9: VI (ff. 96-99, 100-103, 104-107).

10: VI (ff. 108-118, 119).

11-12: VI (ff. 120-131, 132-143).

13: III (ff. 144-149).

14-24: VI (ff. 150-161, 162-173, 174-185, 186-197, 198-209, 210-221, 222-233, 234-245, 246-257, 258-269, 270-281).

25: VI (ff. 282-293).

26: VI (ff. 294-305).

27: I (f. 306) incompleto, falta el final del comentario a Crónicas. [Dos bifolios + un folio suelto de guardas finales].

El manuscrito mantiene la foliación original en números arábigos, correspondiente a la época en que el códice estaba completo. El primer folio de la parte conservada en el Museo Lázaro Galdiano aparece con el número 67, y corresponde al fol. 1 del manuscrito completo, pudiéndose apreciar con claridad que la numeración ha sido corregida ${ }^{7}$. La indicación de parašá o del libro bíblico correspondiente escrita al principio de cada cuaderno, puede considerarse la única indicación de cuaderno. Si hubo otra, ha sido cortada al encuadernar el manuscrito, aunque no es probable dado que la parte del códice conservada en Sevilla tampoco la tiene. Se aprecia el punteado en la parte externa de cada folio y el pautado a punta seca, más visible en el verso.

Este códice en su conjunto, como ya se ha venido señalando en otras ocasiones, presenta una serie de características muy determinadas. Por un lado, no tiene colofón, por lo que no sabemos la fecha exacta de composición, pero está generalmente aceptado que es del siglo XIII. Por su escritura, está claro que es de factura asquenazí; probablemente el escriba procedía del norte de Francia, donde la obra de Raši era muy conocida y difundida. Se trata de una letra semicursiva muy cuidada. Está escrito a dos columnas en la mayoría de las páginas, de 47 líneas cada una. Solamente se escriben a línea tirada los fragmentos que se encuentran en páginas que reproducen ciertos tipos de decoraciones, como ocurre en los fols. 100v (final de Deut) o 284v (final de Esdras), o en los folios en los que se incluyen diagramas, que algunos autores identifican con un mapa ${ }^{8}$. Estos diagramas aparecen en dos ocasiones:

\footnotetext{
${ }^{6}$ Agradezco esta información al Dr. Javier del Barco, quien pudo consultar personalmente el manuscrito de la Biblioteca Colombina.

${ }^{7}$ Véase E. GUTWIRTH, « Cruzando sacras fronteras: sobre el manuscrito de Rashi », Revista de Arte 322, enero-marzo, (2008), p. 75, nota 7.

${ }^{8}$ Cantera se refiere a él como 'cuadro geográfico' mientras que Gurwirth lo califica de 'mapa' (véanse los dos artículos citados). Este último autor se basa
} 
en Num 34, 3-12 y en Ez 45, 1-8 y Ez 48. En el primer pasaje se dan una serie de nombres geográficos de lugares, regiones y límites de Israel dispuestos dentro de varios espacios rectangulares?. En el segundo, se citan las posesiones de los sacerdotes, levitas, la ciudad y los príncipes y al final del libro de Ezequiel se especifica la distribución del territorio. Más adelante daremos más detalles de estos diagramas, referidos a sus decoraciones.

Sabemos que el manuscrito completo se encontraba ya en el siglo XVIII en Sevilla, y que ya le faltaban algunos folios donde podría haber estado el colofón. Pérez Bayer se refiere a él en su Viaje arqueológico desde Valencia a Andalucía y Portugal. El martes 8 de octubre de 1782 refiere el autor su visita a la biblioteca de la «santa iglesia». El canónigo magistral, Don Marcelo Daye, le enseña algunos de los tesoros, entre ellos, dos manuscritos hebreos: uno es una «insigne Biblia Hebrea», añadiendo que fue donado por el rey Alfonso el Sabio a la catedral de Sevilla, así como que a este rey se lo había regalado San Luis, rey de Francia ${ }^{10}$. Y el otro, un «libro Rabino sin puntos, uno y otro excelentemente escritos en pergamino», refiriéndose sin duda a este ejemplar. Lo describe en los siguientes términos:

«...contiene la exposición de la Biblia de R. Salomón Iarchi, esto es, Lunar, por ser, como algunos quieren, natural de Puente Lunel, en Lenguadoc. A este laman comúnmente los hebreos Raschi, y otros Rasi, una y otra dicción compuesta de las iniciales de Rabí Salomón Iarchi, esto es, ש $ר$, ...Su carácter, digo el del códice, es rabino perfectísimo, y tan igual que sorprende, y lo mismo parece una línea que otra, y lo mismo es la tinta. Fáltale al fin alguna hoja (pocas) en que tal vez se hallaría el nombre del escritor, el lugar y el año donde y en que se escribió. En la portada, por bajo de un cuadrilongo o apaisado escrito en caracteres arábigos trabados y cruzados entre sí con mil rasgos y gallardías de pluma y floreados los espacios entre línea y línea con un trabajo verdaderamente ímprobo, hay dos círculos escritos en letra hebrea cuadrada con puntos, la cual, en mi juicio, es posterior al año mil cuatrocientos de Jesucristo.»

A continuación, copia el texto en hebreo. Y añade:

en su similitud con otros mapas de Raši en otros manuscritos y en la terminología ya utilizada por otros autores.

${ }^{9}$ Existen mapas o diagramas similares en muchas copias de comentarios de Raši. Por poner algún ejemplo, citaremos algunos de la BnF (Heb. 154, 155, 156, 161), British Library (Harley 5708), Biblioteca estatal de Baviera en Munich (Cod. Heb. 5), o Vaticana (Vat. Ebr. 94).

10 Algunos fragmentos de este texto de Pérez Bayer han sido citados por todos los autores que han escrito sobre este manuscrito, como los ya mencionados. 
«Parece que el que escribió esto no guardó la ortografía ni aún la propiedad de la escritura y sintaxis

hebrea...»

A partir de este texto podemos deducir dos hechos importantes para este trabajo: por un lado, nos da un indicio más de la trayectoria de la Biblia, antes de terminar en Sevilla ${ }^{11}$. Bien podemos dar como seguro que ambos manuscritos, la Biblia y el comentario de Raši, vinieran juntos, lo que nos corroboraría la procedencia inicial de su escritura: Francia. Y, por otro lado, vemos que en ese momento el manuscrito se encontraba completo en Sevilla, ya que hace alusión al folio $1 \mathrm{r}$ (que describimos a continuación) con su decoración y nota en hebreo sobre su composición. Solamente faltaban, como dice, algunos folios que corresponden con toda seguridad a los que siguen faltando actualmente en el último cuaderno del manuscrito, donde se supone que debía estar el nombre del copista.

\section{Museo Lázaro Galdiano}

Esta porción del manuscrito comprende los siguientes folios: 1, 68, 84 a 95, 100 a 103, 119 y 282 a 293. Hemos aludido antes a una corrección en la numeración del manuscrito, que corresponde al folio 1. Actualmente tiene el número 67, pero era originariamente el primero y estaba numerado con el número uno. La persona que cambió la numeración no debía saber hebreo, ya que se limitó a poner el número anterior al primer folio numerado que tenía delante, en este caso el 68. Dicho folio constituye la portada y, contiene una ornamentación de clara procedencia andalusí. Un marco rectangular que comprende toda la página encuadra dos arcos de herradura muy cerrados con un círculo interior. Bajo estos dos círculos, otro recuadro rectangular enmarca cuatro líneas de escritura cursiva hebrea de carácter arabizante donde se da el título del libro y su autor y, bajo este rectángulo se insertan otros dos arcos lobulados. En su interior se inscribe un texto en

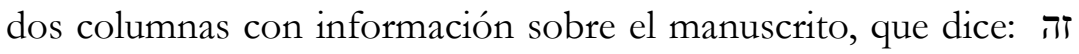
פירוש רבינו שלמה ז"ל צרפתי הכתוב למעלה בכתיבה משרקי הוא זה הספר

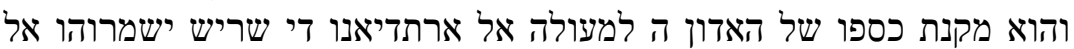
El texto hebreo continúa en el folio siguiente. Toda la decoración de este folio es de marcado tipo andalusí, tanto por sus formas geométricas en los enre-

11 Actualmente, no consta ninguna biblia manuscrita hebrea en el catálogo de la Biblioteca Colombina.

12 «Lo escrito arriba en escritura misraquí es: este libro es un comentario de Rabenu Salomón, bendita sea su memoria, Șarfatí, y fue comprado con el dinero del muy ilustre señor arcediano de Jerez, que Dios le guarde, amén. Y él ordenó ensalzar este libro como él...». 
jados inscritos en los círculos, como por los colores, las cenefas, su diseño y especialmente en la letra cursiva mencionada. De hecho, encontramos diseños muy similares en muchos manuscritos árabes o aljamiados escritos en la Península Ibérica a partir del siglo XV. Los marcos exteriores, como los motivos que aparecen en el margen izquierdo y las decoraciones florales de los cuatro vértices, se pueden encontrar con frecuencia ${ }^{13}$.

En el resto de esta porción del manuscrito abundan las iluminaciones. Tenemos varios ejemplos. En el fol. 85v, la decoración de principio del libro de Deuteronomio en אלה הדברים con la indicación de la parašá 44 aparece, a la derecha del panel ornamental sobre esas dos primeras palabras y entre las dos columnas de texto, un elemento con forma de granada y motivos florales andalusíes en rojo, verde y ocre. Lo mismo ocurre en el fol. 68r (2r), que también incluye motivos de granada en dorado, con perfiles en verde y rojo.

En otros folios los inicios de la decoración solamente se iluminan en rojo, pero con el mismo estilo: 92r שופטים parašá 48; 93v parašá 49; 95r parašá 50. En el fol. 285r, que contiene el principio del comentario a Crónicas, volvemos a encontrar la decoración en rojo y verde.

Hay ocasiones en las que la iluminación ocupa más de medio folio, reproduciendo motivos de igual estilo andalusí. Los motivos entrelazados densos y complicados son típicos de la decoración mudéjar tardía, y son claro reflejo de las decoraciones de las páginas tapiz de los manuscritos árabes. Tenemos ejemplos en el fol.101r, al principio del libro de Josué, donde la ornamentación se ilumina en rojo y dorado, formando paneles en las dos palabras iniciales del libro y en el título del mismo, así como unas cenefas con motivos geométricos de entrelazados en ángulo recto sobre la parte superior de la columna derecha del texto y entre las dos columnas. Se añade un motivo floral exento en forma de granada en el margen exterior, iluminada en verde y rojo. O en el fol.119r al principio de Reyes: en este caso el motivo es ligeramente distinto en estilo a los anteriores, introduciendo nuevos y complicados diseños e incluyendo colores gris y rojo. La palabra מלכים aparece escrita en dorado e inscrita en la viñeta.

También el fol. 100v (22v), al final del comentario a Deuteronomio, reproduce un motivo andalusí de más de media página, aunque en esta ocasión se dibuja exento, no inscrito en rectángulo como en otras ocasiones. La decoración que aparece en el fol. $284 \mathrm{v}$ corresponde al mismo estilo: se trata de un círculo inscrito en un rectángulo que corresponde al final del libro de Esdras. En la parte inferior aparece como decoración una imitación de letras árabes de tipo cúfico en sepia ${ }^{14}$. La decoración que aparece en el

13 Véanse algunos de los manuscritos recogidos en la serie http://manuscripta.bibliotecas.csic.es como el Junta 56 o el Junta 47.

${ }^{14}$ Estos motivos de origen oriental, pero muy adaptados a los modelos de Al-Ándalus, son recurrentes en manuscritos copiados en la Península Ibérica a 
margen derecho guarda similitudes con las reproducidas en Coranes, sobre todo de los procedentes del Magreb o de la Península Ibérica, imitando el cierre de las encuadernaciones de carpeta típicas de estos manuscritos árabes ${ }^{15}$.

En los fols. 84 r y v aparecen los 'mapas' a los que más arriba nos referíamos. En el fol. $84 \mathrm{v}$ el color rojo aparece en dos tonos: uno muy vivo y otro mucho más apagado. El más vivo rodea con una línea al resto, estando el texto central dentro de un recuadro. En el fol. 84r hay un motivo rectangular de entrelazado en rojo y negro que parece hecho posteriormente de forma independiente, tanto por la tinta como por el motivo. Sobre la disposición de los textos de estos mapas, no voy a añadir más a lo ya descrito por E. Gutwirth. Pero sí debo señalar que su similitud con el manuscrito de la BnF Heb. 154 es casi total: la información del diagrama y la disposición de los textos hebreos en él son casi idénticas ${ }^{16}$. Es decir, la diferencia esencial entre los dos radica, sobre todo, en la decoración del manuscrito del museo Lázaro Galdiano. Sin embargo, si comparamos estos mapas con los que aparecen en el manuscrito de Munich (Bayerische Staatsbibliothek, Heb 5) fechado en 1233, comprobamos que sus textos presentan algunas variantes, faltando algunas de las palabras en el de $\mathrm{Madrid}^{17}$.

En otras partes del manuscrito, como en el fol. 88r, en la segunda columna aparece una decoración muy frecuente en otros manuscritos sefardíes de los siglos XIV y XV, aunque de estilo más sencillo, como puede ser el G-II-8 de El Escorial ${ }^{18}$ o incluso

partir del siglo XIII. Podemos encontrar numerosos ejemplos, como en el manuscrito Heb 22 de la BnF, copiado en Castilla en el siglo XIII, o en el Heb 32 de la misma biblioteca, de principios del siglo XV.

15 También conocidas como "pages fermoir", este modelo de decoración ya es usual en Coranes orientales a partir del siglo X, sobre todo. Tenemos ejemplos de estas decoraciones en varios ejemplares, como un Corán de la Biblioteca Real de Marrakesh, manuscrito $n^{\circ} 4$, fols. 4v y 5, copiado en el siglo XIV; en el Corán MS A 6755 de la Biblioteca de la Universidad de Estambul, fol. 3r, copiado en Córdoba en 1143 o en el no 25 de los manuscritos de la JAE de la biblioteca Tomás Navarro Tomás (CSIC), fol. 1v y especialmente en un Corán copiado en el Magreb en 1326, el manuscrito 1740 de la biblioteca de El Escorial, fol. $1 \mathrm{v}$.

${ }^{16}$ En el fol.84v hay una variante textual con respecto al manuscrito de la $\mathrm{BnF}$, después del texto situado en el centro del diagrama, acerca de la montaña Hor, al final, tras כמו תפוח, se añade: קטן על גבי תפוח גדול.

${ }^{17}$ Los mapas de este manuscrito de Munich han sido detalladamente descritos en B. NARKISS, "Rashi's Maps », in E. SCHILlER (ed.), Zev's Vilnay Jubilee Volume, Jerusalem, 1984, p. 435-439 (en hebreo). En este trabajo no se cita el códice conservado en España. Tampoco se cita en ninguno de los tres estudios de Gruber sobre los diagramas que aparecen en este comentario: M.I. GRUBER, «What happened to Rashi’s Pictures? », Bodleian Library Record, 14, 2 (1992), p. 111-124; "Light on Rashi's Diagrams from the Asher Library of Spertus College of Judaica », The Solomon Goldman Lectures 6 (1993), p. 73-85; y « Notes on the Diagrams in Rashi's Commentary to the Book of Kings ", Studies in Bibliography and Booklore 19 (1994), p. 29-41.

18 Para ver una descripción de esta excelente biblia y detalles sobre su texto e iluminaciones, véase F.J. DEL BARCO DEL BARCO, Catálogo de Manuscritos Hebreos de la Comunidad de Madrid, vol. 1, Madrid, 2003, p. 124-126 y M.J. DE 
la Biblia de Kennicott (Bodleian Library, Oxford, Kenn. 1). En este caso se señala la parašá 46, en el libro del Deuteronomio (Deut 7,12), con las palabras הסלת ואתחנן והיה עקב. Lo mismo ocurre en el fol.89v, en la parašá 47: en la decoración predominan los tonos rojo y violeta y se trata de unos paneles enmarcando las palabras y con una serie de prolongaciones hacia abajo o hacia arriba, de trazos muy finos ${ }^{19}$.

\section{Biblioteca Colombina de Sevilla}

La parte del manuscrito conservada en la Biblioteca Colombina de Sevilla no es continuación del anterior. Muy pocos autores lo citan o describen sus características. T. Metzger se refiere a él de forma muy sucinta y tampoco aparece reseñado en la lista editada por Blondheim de manuscritos que reproducen comentarios bíblicos de Rashi ${ }^{20}$. Comienza esta parte del códice con el texto que sigue al primer folio de texto de la parte conservada en el Lázaro Galdiano. La primera parašá que aparece es נח (Gen 6,9). Parece claro que hay partes del manuscrito de Sevilla que fueron llevadas a Madrid, escogidas basándose fundamentalmente en las decoraciones ${ }^{21} \mathrm{y}$, una vez allí, fueron encuadernadas formando un volumen y sin importar la continuidad del texto, dando lugar a que el comentario no pueda leerse de forma seguida, ni en un ejemplar ni en otro. El hecho de que la parte del museo madrileño haya sido numerada correlativamente y por separado da lugar a nuevas equivocaciones en el orden. Sin embargo, esto nos ayuda a comprobar que, tanto la decoración de la parte sevillana como la de la madrileña, corresponden a la misma mano del mismo artista.

Los fols. $185 \mathrm{v}$ y $188 \mathrm{r}$ contienen sendos diagramas sobre las posesiones de los sacerdotes, levitas, la ciudad y los príncipes así como la distribución del territorio en el templo. El primero ocupa

AzCÁRraga SERVERT et al., El Manuscrito Hebreo Bíblico G-II-8 de la Biblioteca de San Lorenzo de El Escorial, Madrid, 2000.

19 Otros manuscritos sefardíes de fines del siglo XIV o del XV presentan este tipo de decoraciones. Por poner algún ejemplo, podemos citar los siguientes: Heb 95 de la BnF, copiado en Castilla a fines del siglo XIV, con decoración hispano mudéjar; Heb 1137 de la $\mathrm{BnF}$, del siglo XV realizado en Provenza o Heb 593 de la BnF, procedente de Marruecos del fines del siglo XV.

20 T. Metzger, «Exégèse de Rashi et Iconographie biblique juive au Moyen Age ", in G. DAHAN - G. NAHON - E. NichOlas (éds.), Rashi et la culture juive en France du Nord au Moyen Age (Collection de la Revue des Etudes Juives, 17), Louvain, 1997, p. 203-221 y D.S. BLONDHEIM, « Liste des manuscrits des Commentaires bibliques de Rashi », Revue des Etudes Juives 91(1931), p. 71-101 y 154-174.

${ }^{21}$ De hecho, la mayoría de las páginas conservadas en Sevilla carecen de decoraciones, si exceptuamos los encabezamientos y los folios 130v, 185v, 188r y $208 \mathrm{v}$, mientras que el primer folio del manuscrito, el más ricamente decorado, se incluye en el bloque del Museo Lázaro Galdiano. Parece que la persona que separó los folios destinados al museo le daba especial valor a estas páginas iluminadas, sin tener en cuenta el texto del comentario. 
un recuadro insertado en la parte inferior de la columna derecha de la escritura, ligeramente fuera de ella y el segundo de ellos ocupa las tres cuartas partes de la página.

El diagrama del fol. 185v inserto en el comentario a Ez. 45 es de forma rectangular, dividido en tres franjas horizontales. La parte central es más ancha y está, a su vez, dividida en otras tres, siendo la izquierda aproximadamente el doble de ancha de las otras dos. Aparecen reseñadas las posesiones de la ciudad, de los levitas, y de los sacerdotes, alrededor de un cuadro central que representa el Har habáyit. La parte superior corresponde a la porción del príncipe y la inferior a la de la ciudad.

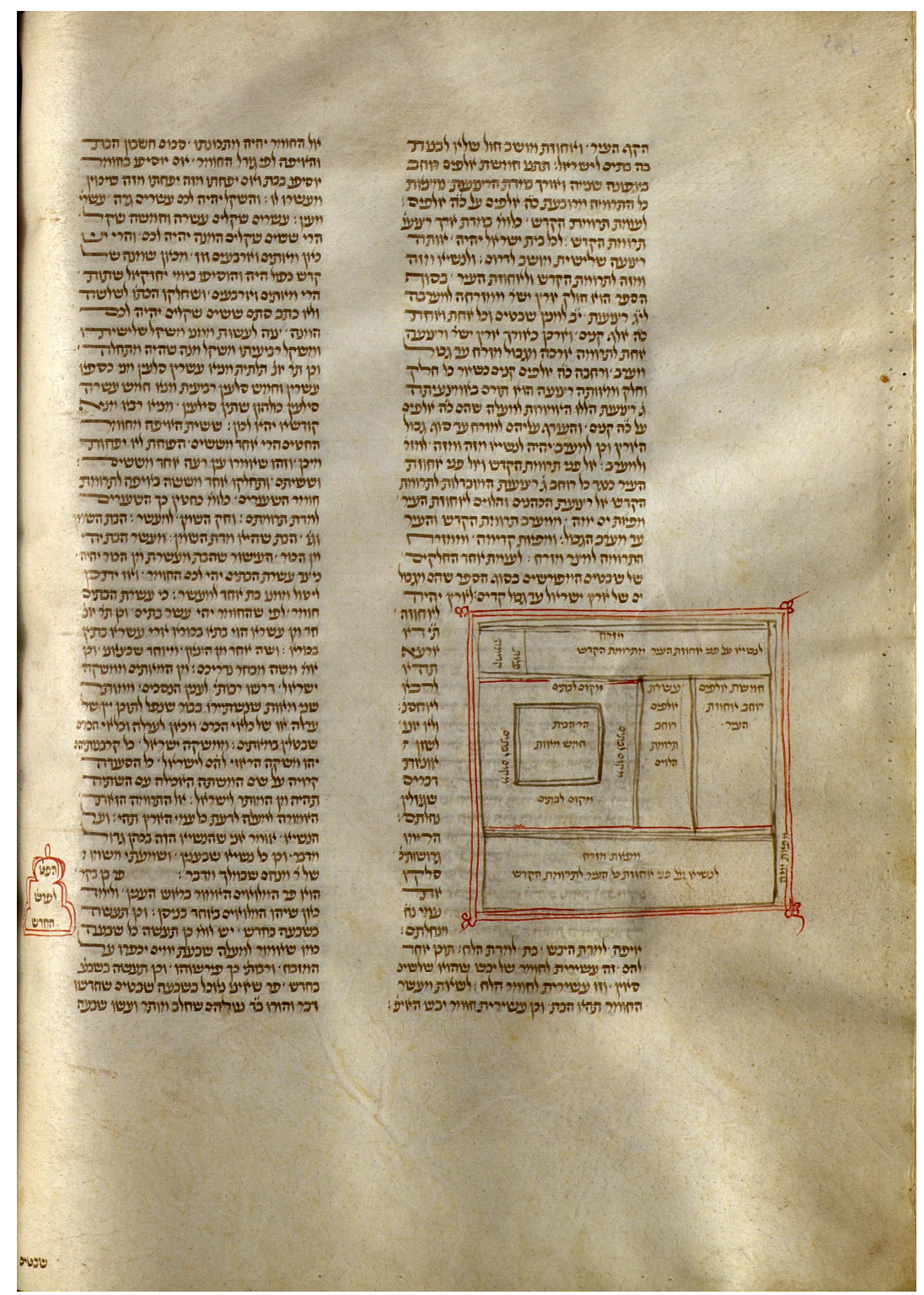

Biblioteca Colombina, ms. 56-1-16, fol. 188r.

El diagrama sobre la distribución del territorio del templo que aparece al final del comentario a Ezequiel, tras el capítulo 48 (fol. 188r), representa el reparto del país entre las doce tribus. Sus nombres se deberían escribir a ambos lados del diagrama, siete a un lado y cinco al otro, según el orden en que aparecen en el texto 
bíblico de Ezequiel, (Ez 48, 1 a 7 y 23 a 27) y como aparecen en el Heb 154 de la BnF. Sin embargo, en este diagrama aparecen Judá, Rubén, Efraim, Manasés, Naftalí y Aser a la izquierda, mientras que Gad se escribe con las otras cinco a la derecha. A la izquierda se inscribe el Har Habayit en un rectángulo con los sacerdotes (kohanim) a los cuatro lados. En la parte central se señala la parte que se reserva como ofrenda y las que se destinan a los sacerdotes; finalmente, en la parte baja del diagrama se especifican los dominios atribuidos a los príncipes (Ez 48, 21). Como en los demás manuscritos, el oriente se sitúa en la parte superior del cuadro. Mientras que en los demás manuscritos los diagramas se dibujan de forma muy rudimentaria, en este caso se rodean de un marco decorado con una cenefa con entrelazados de influencia mudéjar.

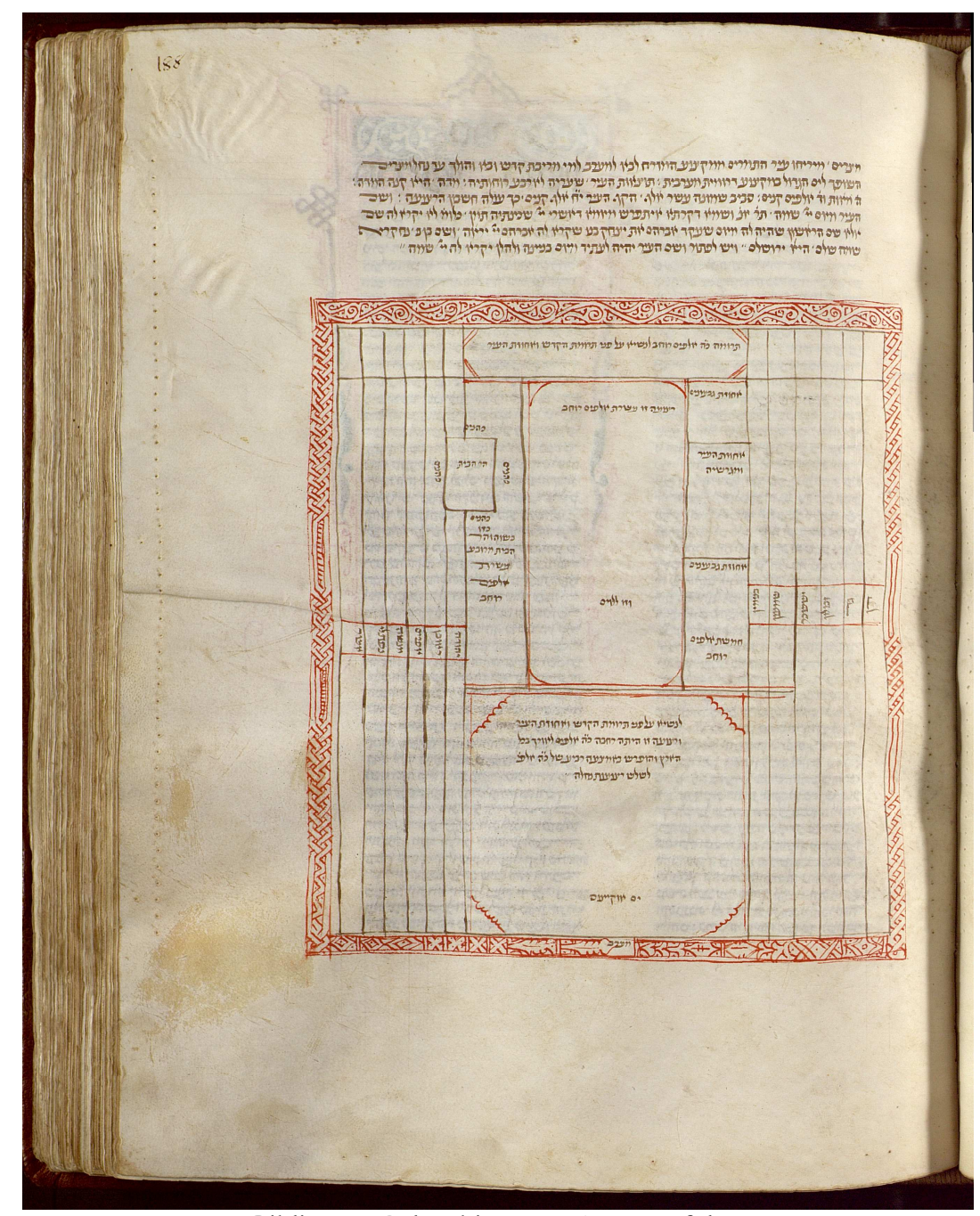

Biblioteca Colombina, ms. 56-1-16, fol. 188r.

T. Metzger encuentra similitudes en la reproducción de los diagramas y en la tinta utilizada del manuscrito de la Biblioteca Colombina con el autor de los 4 mapas que se reproducen en los manuscritos Heb 154, Heb 156 (ambos de la BnF), Harley 5708 (de la British Library) y Cod. Heb 220 (de la Österreichische Na- 
tional Bibliothek de Viena) ${ }^{22}$. Heb 154 está fechado en 1298 y los demás son coetáneos. A estos hechos se suma la afirmación de G. Sed-Rajna ${ }^{23}$, acerca de que los cuatro diagramas principales de Raši (los que ilustran los libros de Números y Ezequiel) aparecen en los manuscritos del siglo XIII o comienzos del XIV, de factura poco sofisticada, con variantes mínimas entre ellos. También afirma que los diagramas que representan el tabernáculo y la menorah no aparecen en copias tempranas. Es decir, este es también el caso del códice que nos ocupa.

Por lo tanto, aceptando estas semejanzas, junto con la similitud entre ambos manuscritos de los diagramas del libro de Números antes aludidos (fols. 84r y v), así como la ausencia de la representación de la escala de Jacob en Génesis 28,12, y la afirmación de T. Metzger de que «existe un manuscrito de finales del siglo XIII o principios del XIV que contiene los dos mapas de Ezequiel» ${ }^{24}$ (como ocurre en nuestro documento),el manuscrito de Madrid y Sevilla debe datarse también a fines del siglo XIII, hecho que contradice la datación más temprana que apunta Gutwirth. Por otra parte, la escritura del códice también es muy similar al Vat. Ebr. 94, datado en el mismo siglo XIII.

El origen de estos diagramas se ha atribuido al propio Raši por los autores ya citados (Metzger, o Sed-Rajna), basándose en una carta suya dirigida a los rabinos de Auxerre y contenida en el manuscrito de la Preussischer Staatsbibliothek de Berlin Or. Fol. 122. A raíz de esa carta, la teoría más aceptada es que estos diagramas ya formaban parte del texto original de Raši y que fueron reproducidos en las distintas copias de sus manuscritos. Los estudiosos también se han basado en el comentario de Rashbam para atribuirlos a Raši ${ }^{25}$. Sin embargo, las ilustraciones más antiguas que existen de estos diagramas son posteriores a la época de Raši, por lo que nos es imposible saber hasta qué punto reproducían las aludidas por él en esa carta. Lo más probable es que no estuvieran siquiera incluidos en los primeros manuscritos de los comentarios de Raši, sino que se hubieran incorporado en las copias más tardías, aunque tampoco aparecen en muchos de los primeros impresos, como sucede con el incunable Heb 94b del Jewish Theological Seminary ${ }^{26}$. Sin embargo, parece que otros ejemplares les die-

${ }^{22}$ Véase T. METZGER, « Exégèse de Rashi et Iconographie biblique juive au Moyen Age », in G. DAHAN - G. NAHON - E. NICHOLAS (eds.), Rashi et la culture juive en France du Nord au Moyen Age, Louvain, 1997, p. 203-221.

23 G. SED-RAjNA, "Some further Data on Rashi's Diagrams to his Commentary on the Bible », Jewish Studies Quarterly 1/2 (1993-94), p. 149-157.

24 T. METZGER, op. cit. p. 211.

25 Rashbam en su comentario a Num. 34,2 y acerca del reparto de la tierra de Israel, escribe: «Mi abuelo, nuestro maestro, lo explicó [este texto] y dibujó los límites». Véase M.I. LOCKSHIN (ed.), Rashbam's commentary on Leviticus and Numbers: an annotated translation, Providence: Brown University, 2001, p. 295. Sin embargo, T. Metzger, en el artículo citado, no le da importancia a este texto en ningún momento.

${ }^{26}$ Impreso en Zamora en la imprenta de Samuel ibn Musa en 1487? Hay distintas teorías sobre su datación, véase S. IAKERSON, Catalogue of Hebrew Incu- 
ron importancia e insertaron algunos de ellos, como ocurre en el Heb94a del mismo JTS (fol. 73r) ${ }^{27}$. En este caso, el diagrama es un dibujo a mano muy tosco y rudimentario, y también reproduce la menorah antes mencionada en el fol. 36v.

Pero tanto si los dibujos estuvieron en aquellas primeras copias o no, no puede ser coincidencia que haya diagramas similares en otros comentarios bíblicos cristianos de la época. Quizás pueda ser que el propio Raši imitara, al introducirlos como explicaciones en sus textos, otros manuscritos anteriores cristianos. En concreto, se le ha vinculado con un comentario a Ezequiel hecho por Richard de San Víctor, monje del siglo XII que llegó a ser prior de la abadía de San Víctor de Paris en 1162, quien hace representaciones similares en varios manuscritos latinos ${ }^{28}$. Los comentaristas cristianos, a partir del siglo XII, enriquecen sus textos en buena medida con las fuentes de la exégesis judía, sobre todo a partir de Raši y la escuela por él creada. La interpretación judía de esta corriente, basada en el sentido literal del texto hebreo de la Biblia y en la gramática y la tradición judía, influye notablemente en muchos autores cristianos. En palabras de G. Dahan, «le début du XII siècle est, à en juger par des nombreux témoignages, une époque d'intenses échanges au sujet de la Bible entre chrétiens et juives» ${ }^{29}$. Precisamente esta época representa un periodo de transición, en el que los comentaristas cristianos recurren con frecuencia a las obras de los comentaristas judíos, y de ello existen numerosos ejemplos ${ }^{30}$. No hay que olvidar que los eruditos dedicados al estudio de la Biblia eran una élite entre las personas de cierto nivel intelectual, muy escasos, y que las corrientes religiosas fluían entre todos ellos de forma natural, más aún si consideramos que todos pertenecían al mismo ámbito geográfico. Por lo tanto, no sería de extrañar que el modelo de unos diagramas explicativos llegara a ser un elemento didáctico importante y hubiera servido para ilustrar varios manuscritos de distintos autores, tanto cristianos como judíos. En cuanto al tipo y a la disposición de los diagramas, algunos autores como B. Kedar afirman que tampoco Raši fue el primero que los ideó, ya que se han encontrado paralelos en otros mapas cristianos, a los que Raši habría tenido acceso, como son el que aparece en la versión latina de San Jerónimo del

nabula from the Collection of the Library of the Jewish Theological Seminary of America, vol. 2. New York - Jerusalem, 2005, no 97, p. 375.

${ }^{27}$ Fechado en 1487 en la imprenta de Soncino. Véase S. IAKERSON, Catalogue of Hebrew Incunabula from the Collection of the Library of the Jewish Theological Seminary of America, vol. 1. New York - Jerusalem, 2005, nº 32, p. 124-127.

28 Véase G. SED-RAjNA, "Some further Data on Rashi's Diagrams to his Commentary on the Bible ", Jewish Studies Quarterly 1/2 (1993-94), p. 149-157. En las páginas 155 a 157 esta autora se refiere en concreto a dos manuscritos de la BnF: lat. 3438 y lat. 1791.

29 G. DAHAN, L'exégèse chrétienne de la Bible en Occident médiéval. XII - XIV siècle. Paris, 1999, p. 102.

${ }^{30}$ Véase, por ejemplo, G. DAHAN, op. cit, especialmente el capítulo III y G. DAHAN, Les intellectuels chrétiens et les juifs au moyen âge, Paris, 1999. 
Onomasticon de Eusebio de Cesarea, o en otros posteriores de los siglos XI y XII ${ }^{31}$.

El manuscrito incluye otros diagramas de menor tamaño y más sencillos, incluidos en un espacio en blanco entre dos líneas de texto. Esto ocurre en Jueces 21,19 (fol. 108v), a propósito de la situación de Šiloh. Aparecen los nombres de Šiloh, Líbano, Betel, Siquem, la calzada y el oriente encuadrados por unos marcos de tres líneas, con trazo muy rudimentario. Estos seis términos se citan en el texto del versículo para situar el enclave. En el fol. $121 \mathrm{r}$, en el comentario a 1 Re 6,31 se dibuja en el margen izquierdo un ángulo con el vértice hacia arriba que representa la entrada al interior del templo de Salomón. Estos y otros motivos aparecen con frecuencia en los manuscritos de los comentarios de Raši, de forma más o menos esquemática, como en este caso; las omisiones de estas ilustraciones más destacadas en este códice son el dibujo de la menorah en Ex 25,31, la escala de Jacob de Gen 28,12, la corona en 1 Re 1,39 y el dibujo del efod en Ex 28, $6^{32}$.

Por último, el folio 208v (BC), al terminar el comentario a Profetas y antes de comenzar el de Hagiógrafos, tiene un motivo decorativo de más de media página con un círculo con entrelazados geométricos, inscrito en un marco con cenefa, en un estilo similar a los anteriores aunque con notables variaciones en su diseño. Da la impresión de que al separar los folios o cuadernos que se llevaron a Madrid se intentó tomar la mayor parte de las iluminaciones principales del manuscrito, sin tener en cuenta para nada, como ya he señalado, la continuidad del texto. De hecho, este folio es el único que queda en Sevilla con decoración significativa, debido quizás a ser el único folio con iluminaciones en el bloque comprendido entre los cuadernos 14 a 24 del códice (66 folios), por lo que pudo fácilmente pasar desapercibido. Otras decoraciones de menor tamaño aparecen al inicio de las parashbiyyot, también con motivos de entrelazados geométricos similares a las ya descritas de influencia mudéjar, como ocurre por ejemplo en los fols. $14 \mathrm{v}$ o $19 \mathrm{r}$. En ocasiones, parece que la decoración se ha hecho sobre un espacio en el que no estaba previsto insertarla al diseñar la página del manuscrito.

${ }^{31}$ Véase B.Z. KEDAR, «Rashi’s Map of the Land of Canaan, ca. 1100, and its cartographic Background », in R.J.A. TALBERT - R.W. Unger (eds.), Cartography in antiquity and the Middle Ages: fresh perspectives, new methods, Leiden, 2008, p. 154-168.

${ }^{32}$ Hay otros motivos menos frecuentes que sólo se reproducen el algún manuscrito, como las uvas de Canaán de Núm. 13,23. 


\section{Conclusión}

Haciendo un repaso sobre la decoración e ilustraciones de este comentario y tras este breve recorrido por todas ellas comparándolas con otros manuscritos similares, podemos afirmar que se aprecian claramente varias etapas en su composición. La primera, es la composición del comentario propiamente dicha. El escriba de origen askenazí lo copia no sabemos exactamente dónde y probablemente a finales del siglo XIII, incluyendo los diagramas explicativos que ya existían en otros comentarios de Raši y que, probablemente, fueron diseñados por él mismo. La segunda etapa corresponde a las iluminaciones. Se realizan como complemento del texto y ya en territorio de Sefarad añadiendo, en los casos de los diagramas, una función decorativa a la primitiva función didáctica: todas ellas son de un estilo andalusí, tanto en los motivos como en las disposiciones. La decoración de las palabras iniciales de los libros bíblicos es una de las características más importantes de las iluminaciones que se hacen en la Península Ibérica, sobre todo desde mediados del siglo XIV y todo el XV, en muchos casos extendiéndose a los márgenes de la página, como ocurre en este manuscrito. También los motivos de entrelazados utilizados son muy característicos de los manuscritos ibéricos de finales del siglo XIV y XV $\mathrm{XV}^{33}$. Se utilizan distintas tintas de colores, y se incluye en la obra una primera página muy ornamentada con un texto indicando que el manuscrito ha sido adquirido por el arcediano de Jerez. Dada la similitud, como hemos apuntado anteriormente, con otros manuscritos sefardíes del XV, se podría pensar en esta fecha aproximada para estas iluminaciones.

Sin embargo, no todas estas ilustraciones se realizan en una sola vez. Como ejemplo, hemos visto que la decoración del folio $84 \mathrm{r}$ tiene un motivo de entrelazado en rojo y negro que puede apreciarse hecho en una fase distinta del resto. Las tintas son mucho más oscuras y los colores mucho más intensos que el resto del cuadro. En esta época, las decoraciones adoptan los usos de las regiones donde se producen, repitiéndose los motivos y utilizando las corrientes estilísticas de las decoraciones de manuscritos cristianos en muchos casos. La utilización en este manuscrito de elementos típicos andalusíes no es de extrañar, teniendo en cuenta tanto la movilidad de los escribas e iluminadores por los territorios de Sefarad, que llevan su estilo a distintas regiones, como la posibilidad de iluminadores que trabajaran tanto en manuscritos hebreos como cristianos, incorporando en muchos casos elementos mudéjares.

33 Véase, por ejemplo, los motivos del manuscrito Parm. 2674 (De Rossi 850) fechado en 1469 o Parm. 677 fechado en 1473, ambos producidos en Lisboa; también el Cod. 37 A 2, una biblia de Monte Olivetto Maggiore, del siglo XIV y el Heb 32 de la BnF copiado en España en el siglo XV. 
Finalmente, tenemos otros detalles que destacan como independientes del resto del manuscrito: aparecen cuatro notas marginales explicativas del texto y que amplían o especifican aspectos del comentario en los fols. 86v, 88r y 88v, es decir, en la parte conservada en Madrid. Todas ellas son de la misma mano, en letra semicursiva sefardí y tinta negra. Este tipo de letra es muy común en manuscritos sefardíes de los siglos XIV y XV que contienen otros comentarios bíblicos ${ }^{34}$. Podemos afirmar que fueron añadidas aproximadamente en la misma época que la decoración. Nos encontramos pues, ante uno más de los ejemplares manuscritos compuestos en varias etapas y en varias regiones distintas, circunstancia especial que nos ha permitido aventurarnos a puntualizar algunos detalles en la historia de su composición.

\section{Bibliografía}

M.J. De AzcÁrRaga Servert et al., El Manuscrito Hebreo Bíblico G-II-8 de la Biblioteca de San Lorenzo de El Escorial, Col. Scriptorium, Madrid, 2000.

F.J. DEL BARCO, Catálogo de Manuscritos Hebreos de la Comunidad de Madrid, 3 vols. Madrid, 2003-2006.

F.J. DEL BARCO, Catálogo de manuscritos hebreos de la Biblioteca de Montserrat, Barcelona, 2008.

D.S. BLONDHEIM, « Liste des manuscrits des Commentaires bibliques de Rashi », Revue des Etudes Juives 91 (1931), p. 71-101 y 154-174.

F. Cantera Burgos, « Nueva serie de manuscritos hebreos en Madrid », Sefarad XIX (1959), p. 36-42.

G. DAHAN, L'exégèse chrétienne de la Bible en Occident médiéval. XII'XIV siècle. Les Éditions du Cerf, Paris, 1999.

G. Dahan, Les intellectuels chrétiens et les juifs au moyen âge, Paris, 1999.

M.I. Gruber, «What happened to Rashi's Pictures? », Bodleian Library Record, 14, 2 (1992), p. 111-124.

M.I. GRUBER, «Light on Rashi's Diagrams from the Asher Library of Spertus College of Judaica », The Solomon Goldman Lectures VI (1993), p. 73-85.

M.I. GRUBER, «Notes on the Diagrams in Rashi's Commentary to the Book of Kings», Studies in Bibliography and Booklore 19 (1994), p. 29-41.

34 Por citar algún ejemplo, el comentario a Génesis y Éxodo de Abraham ibn Ezra, manuscrito $\mathrm{n}^{\circ} 8$ de la Biblioteca de la Universidad Complutense de Madrid, del siglo XV, el Sefer ha-Miǩlol de David Qimhi, manuscrito n 20 de la misma biblioteca, también del siglo XV, o el manuscrito 5467, fechado en 1348 que copia el comentario a Profetas Anteriores de David Qimhi. 
E. GUTWIRTH, "Cruzando sacras fronteras: sobre el manuscrito de Rashi », Revista de Arte 322, enero-marzo (2008), p. 67-76.

S. IAKERSON, Catalogue of Hebrew Incunabula from the Collection of the Library of the Jewish Theological Seminary of America, New York Jerusalem, 2005.

B.Z. KEDAR, "Rashi's Map of the Land of Canaan, ca. 1100, and its cartographic Background ", in R.J.A. TALBERT - R.W. Unger (eds.), Cartography in antiquity and the Middle Ages: fresh perspectives, new methods, Leiden, 2008, p. 154-168.

M.I. LOCKSHIN (ed.), Rashbam's commentary on Leviticus and Numbers: an annotated translation, Providence: Brown University, 2001.

T. MetzGER, « Exégèse de Rashi et Iconographie biblique juive au Moyen Age ", in G. Dahan - G. Nahon - E. Nicholas (eds.), Rashi et la culture juive en France du Nord au Moyen Age (Collection de la Revue des Etudes Juives 17), Louvain, 1997, p. 203-221.

B. NARKIss, « Rashi's Maps », in E. SCHILLER (ed.), Zev's Vilnay Jubilee Volume, Jerusalem (1984), p. 435-439 (en hebreo).

M.T. Ortega-Monasterio, " Hebrew Collections in Spain: Catalogues and Inventories ", in J.P. MONFERRER-SALA H.G.B. Teule - S. TORAllas TOVAR (eds.), Eastern Christians and their Written Heritage (Eastern Christian Studies 14) Louvain, 2012.

M.T. ORTEGA-MONASTERIO, « Historia y formación de las colecciones de manuscritos hebreos en España », in E. ALFONSO J. Del Barco - M.T. Ortega Monasterio - A. Prats (eds.), Biblias de Sefarad, Madrid, 2012, p. 149-175.

G. SED-RAJNA, «Some further Data on Rashi's Diagrams to his Commentary on the Bible ", Jewish Studies Quarterly 1/2 (199394), p. 149-157.

RESUMEN - Existen numerosos manuscritos hebreo que copian el comentario de Rashi a la Biblia. Pero hay un ejemplar en España que reúne unas características especiales. El códice se encuentra dividido en dos fragmentos conservados en dos bibliotecas distintas: la del museo Lázaro Galdiano de Madrid y la Biblioteca Colombina de Sevilla. Un estudio sistemático de ambos fragmentos al mismo tiempo y la comparación de varias de sus decoraciones e ilustraciones con otros manuscritos similares permiten hacer algunas puntualizaciones a los estudios parciales ya hechos sobre el códice, realizado en varias fases y en épocas distintas, reflejo de diversas tradiciones culturales existentes en la Europa judía medieval. 
ABSTRACT - An important number of manuscripts reproduce Rashi's commentary on the Torah. Among them, we can found in Spain a codex with particular characteristics: the codex is divided in two parts kept in two different libraries: the Lázaro Galdiano museum in Madrid and the Colombina Library in Seville. A systematic study of both fragments at one side and the comparison between its decorations and illuminations with those of other similar manuscripts allows us to make some considerations and remarks to the partial studies published until now. The codex, written in different stages and different periods, reflects diverse cultural traditions existing in Iberian Peninsula during the Middle Ages.

Palabras Clave - Manuscritos hebreos, codicología, comentarios bíblicos

KeY Words - Hebrew Manuscripts, Codicology, Biblical commentaries 